\title{
New application of myocardial antimyosin scintigraphy: diagnosis of myocardial disease in polymyositis
}

Dominique Le Guludec, Françoise Lhote, Pierre Weinmann, Isabelle Royer, Bernard Jarrousse, Nadine Caillat-Vigneron, Loïc Guillevin, Jean-Luc Moretti

\begin{abstract}
Heart disease is a rare but important complication of polymyositis. Diagnosis of myocardial disease is usually based on non-specific clinical, electrocardiographic, and echocardiographic data. This paper reports a case of polymyositis with myocardial disease diagnosed by myocardial imaging with radiolabelled antibody to myosin, a specific marker of the necrotic myocardial fibre.
\end{abstract}

(Ann Rheum Dis 1993; 52: 235-238)

Polymyositis is an inflammatory disorder of the skeletal muscles that may affect the heart. ${ }^{1}$ Diagnosis of myocardial disease may be difficult in the absence of specific criteria. Endomyocardial biopsy is an invasive diagnostic method and has rarely been reported. In the absence of coronary artery disease, an abnormal resting thallium-201 myocardial scan is considered to be consistent with myocardial disease. ${ }^{2}$ This observation suggests the potential diagnostic value of a scintigraphic scan using a radiolabelled antibody to myosin, which is a specific marker of necrotic myocardial fibres.

\section{Case report}

A 67 year old white man was admitted to hospital in May 1989 with acute pulmonary oedema. In February 1989 he was first seen for progressive dyspnoea, a $14 \mathrm{~kg}$ weight loss, proximal muscle weakness, and typical Raynaud's phenomenon. His chest radiograph showed a diffuse reticular pattern, confirmed by a pulmonary computed tomography scan; the electrocardiogram was normal. Analysis of arterial blood gases showed partial arterial oxygen pressure $\left(\mathrm{PaO}_{2}\right) 92 \mathrm{mmHg}$, partial arterial carbon dioxide pressure $\left(\mathrm{PaCO}_{2}\right)$ 28.5 $\mathrm{mmHg}$, and $\mathrm{pH} 7 \cdot 37$. Pulmonary function studies showed restrictive ventilatory impairment. The creatine kinase was $7125 \mathrm{IU} / \mathrm{l}$ (normal < $170 \mathrm{IU} /$ ) with an MB fraction of $2 \cdot 8 \%$. Rheumatoid factor, antinuclear, antiDNA, anti-PMl, and anti-JOl antibodies were not detected. An electromyogram showed a typical myopathic pattern and a skeletal muscle biopsy sample showed severe degeneration of fibres with discrete perivascular mononuclear cell infiltration. The diagnosis was polymyositis with diffuse interstitial pneumonitis.
The patient was treated with prednisone $(0.5$ $\mathrm{mg} / \mathrm{kg} /$ day) with a monthly pulse of cyclophosphamide and an initial clinical improvement was observed.

Three months later physical examination showed an acutely ill, tachypnoeic man with orthopnoea and diffuse crackles in both pulmonary fields. His chest radiograph showed vascular redistribution to the upper lobes, with diffuse alveolar opacities. The electrocardiogram showed diffuse inversion of the $T$ waves and intermittent atrial flutter. Analysis of arterial blood gases performed under 9 1/minute nasal oxygenotherapy showed $\mathrm{PaO}_{2} 79$ $\mathrm{mmHg}, \mathrm{PaCO}_{2} 31 \mathrm{mmHg}$ and $\mathrm{pH} \mathrm{7} \cdot 31$. The creatine kinase was $815 \mathrm{IU} / 1$ with an $\mathrm{MB}$ fraction of $15 \cdot 4 \%$. Symptomatic treatment with frusemide and intravenous nitrates led to a rapid clinical improvement.

Myocardial thallium tomoscintigraphy was performed one week after the clearance of pulmonary oedema. Acquisition over $180^{\circ}$ was performed at rest, 10 minutes, and three hours after the injection of $103.6 \mathrm{MBq}$ of thallium201. The first images showed an inferior defect (fig 1). Two days later, $74 \mathrm{MBq}$ of antimyosinindium-DTPA (Myocint Centocor) were injected. Planar scintigraphic images were obtained at 48 hours with a $20 \%$ window centred on 173 and $247 \mathrm{KeV}$ photopeaks of indium-111 and a preset time of 10 minutes. Three views were acquired: anterior, $40^{\circ}$ left anterior oblique, and left lateral. They showed a diffuse myocardial uptake affecting the whole myocardium, with an inferior predominance. This abnormal uptake was seen in all three views (fig 2). On the anterior view, the cardiac area was enlarged. The ratio between cardiac activity and lung activity for each pixel on manually selected regions of interest (avoiding the liver and the sternum) was calculated as $2 \cdot 1$ for the total heart and 2.55 for the inferior wall. These ratios were markedly greater than those of our laboratory controls $(1.54(0.15))$. The patient was diagnosed as having diffuse myocarditis with the inferior wall markedly affected. A total body scan did not show any abnormal uptake in the peripheral muscles in spite of active skeletal muscle disease. Four weeks after the pulmonary oedema was detected a coronary and left ventricle angiography showed normal coronary arteries, and inferior wall hypokinesia with normal left ventricular ejection fraction $(72 \cdot 4 \%)$. An endomyocardial biopsy of the right ventricular 

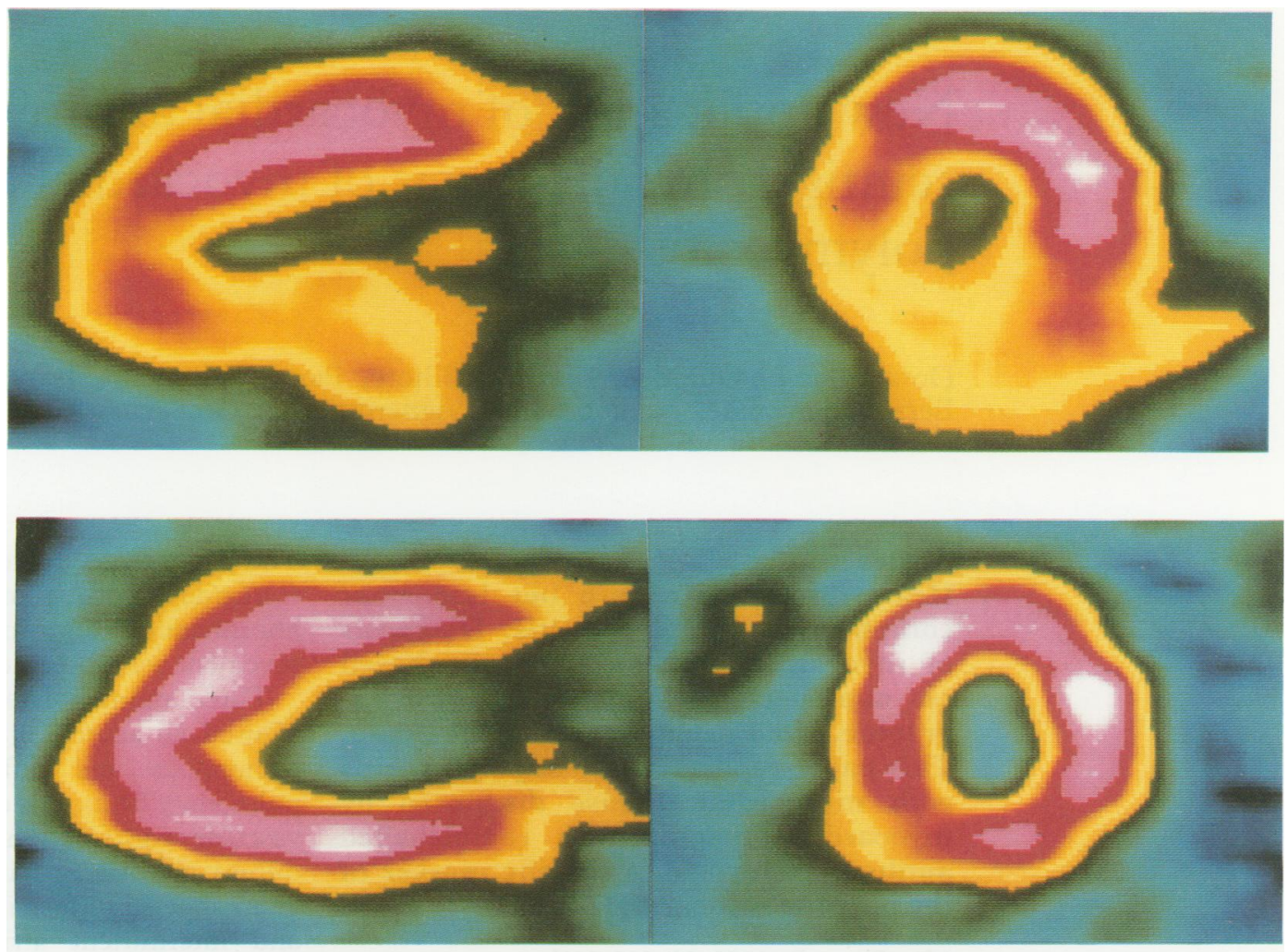

Long axis

Short axis

Figure 1 Thallium tomography at rest. (Upper figures) Initial scan during the acute cardiac episode showing the inferior defect. (Lower figures) Second scan, three months after the start of treatment, showing the disappearance of the inferior defect.

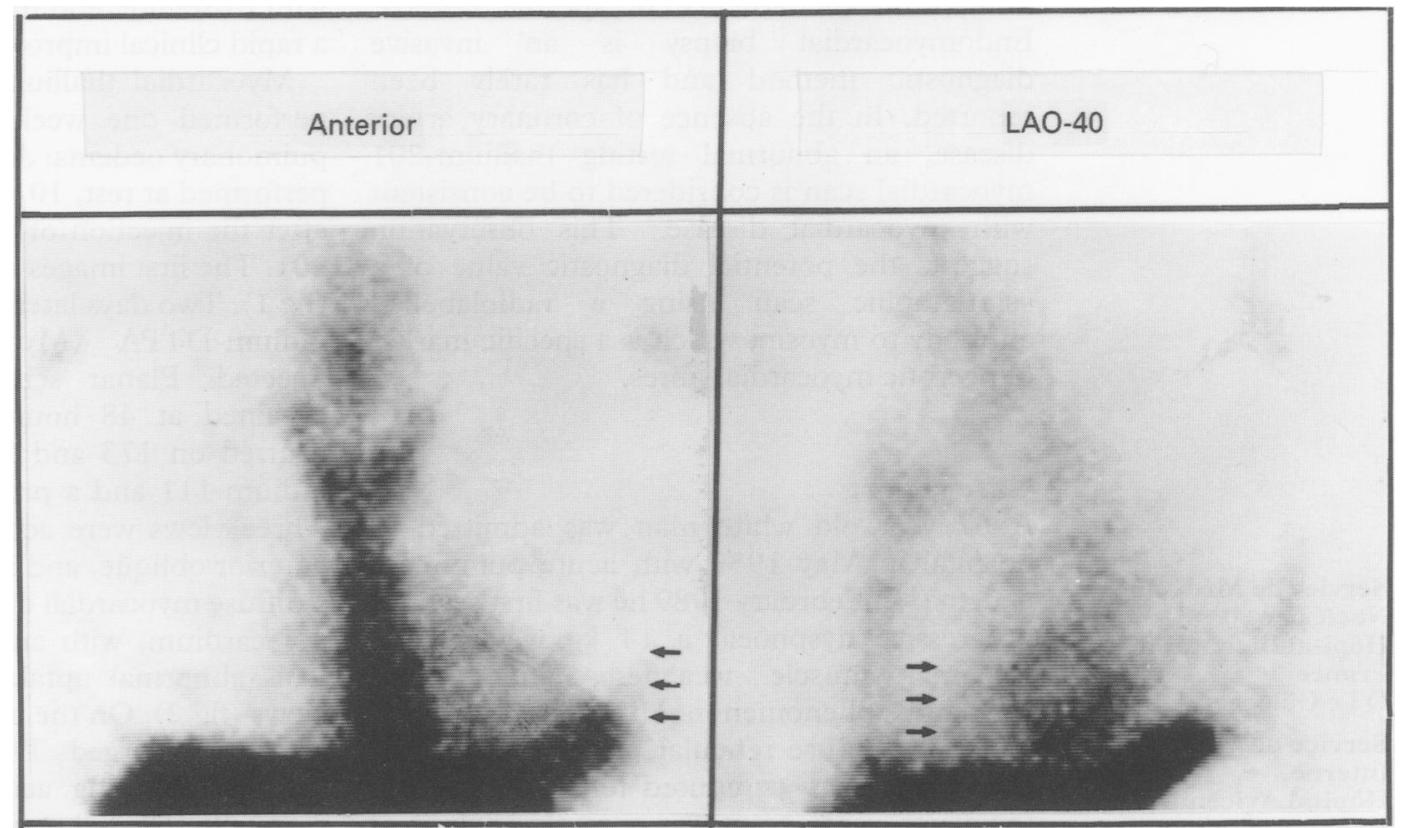

Figure 2 Indium antimyosin planar scans in two views: anterior and $40^{\circ}$ left anterior oblique (LAO). Initial scans during the acute cardiac episode showing the abnormal cardiac uptake.

septum showed perivascular interstitial fibrosis. By continuing treatment with prednisone and cyclophosphamide a long lasting clinical improvement was obtained with normalisation of serum muscle enzymes and the electrocardiogram. In September 1989 thallium and antimyosin scintigraphies were repeated: the rest thallium images were normal, as were the antimyosin images which showed the disappearance of the cardiac uptake and a normal heart/lung ratio of 1.61 (fig 3).

\section{Discussion}

Cardiac disease in polymyositis/dermatomyositis is suspected on electrocardiographic changes such as non-specific ST-T changes, ${ }^{1}{ }^{2}$ variable degrees of atrioventricular block, and bundle branch blocks ${ }^{3}$; cardiac arrhythmias, congestive heart failure or hyperkinetic left ventricular function, ${ }^{4}$ and pericarditis ${ }^{5}$ have been reported. Echocardiography and, more rarely, perfusion scintigraphy with thallium201 or radionuclide ventriculography may be of interest. The increased MB fraction of 


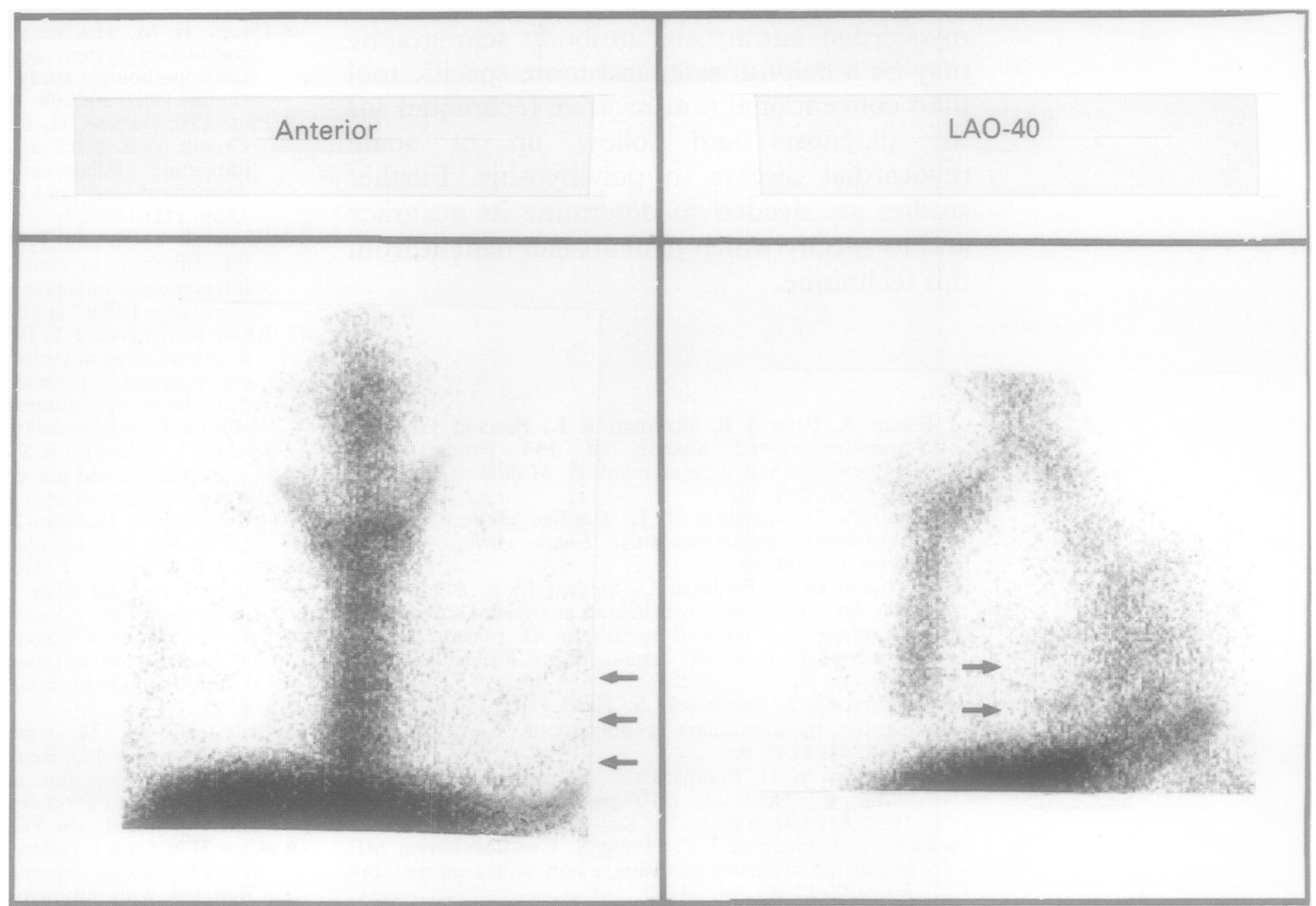

Figure 3 Indium-antimyosin planar scans. Second scans three months after start of treatment showing the disappearance of the abnormal cardiac uptake.

creatine kinase isoenzymes is not correlated with the presence of cardiac abnormalities. ${ }^{6}$

The incidence of cardiac disease in polymyositis/dermatomyositis was thought to be low but has considerably increased with careful cardiac evaluation. ${ }^{24} 78$ This incidence was reported as being from $37 \% \%^{3}$ to $49 \%{ }^{5}$ in large series. Congestive heart failure is reported more rarely: $3.3 \%$ in the 153 patients studied by Bohan et al ${ }^{1}$ and $2.8 \%$ in another large series. $^{5}$

Histological examination of the heart in patients with polymyositis at necropsy showed myocardial changes similar to those seen in skeletal muscles: muscle fibre degeneration and necrosis, focal fibrosis, and varying degrees of interstitial and perivascular mononuclear cell infiltration. ${ }^{4}$ In a clinicopathological study of 16 patients at necropsy reported by Haupt and Hutchins, ${ }^{8}$ microscopic alterations were present in four patients (25\%).

The implication of cardiac disease as the cause of death in patients with polymyositis has been assessed with various results. ${ }^{679}$ In the 76 patients studied by Hochberg et al, ${ }^{3}$ cardiac disease was the major prognostic factor.

Our patient's diagnosis was based on clinical features, but was strongly supported by myocardial scintigraphy. In a previous report, ${ }^{2}$ a thallium scan showed a large defect in the uptake when polymyositic myocardial disease was present. The antimyosin-indium-DTPA antibody (Myocint Centocor) is an $F(a b)_{2}{ }_{2}$ fragment of a monoclonal murine antibody, specific to myocardial myosin. The linkage to DTPA modifies the charge of the antibody and decreases the non-specific uptake, and thus facilitates its diffusion into the necrotic area. Antimyosin antibody specifically binds to cells that have lost their membrane integrity, thus exposing intracellular myosin to extracellular fluid. ${ }^{10-12}$ The binding of the antibody to myofibrils through sarcolemmal holes has been shown by electron microscopy examination of dead myocytes. The antimyosin antibody was labelled with technetium-99m or indium-111 for scintigraphic use. The radiolabelled $\mathrm{F}(\mathrm{ab})_{2}{ }_{2}$ fragment of the antibody appears not to be immunogenic when administered intravenously, has a relatively short blood pool half life, and a suitable dosimetry for clinical studies. Antimyosin antibody scintigraphy has been used to localise and quantify areas of myocardial necrosis. In canine models a close correlation between the scintigraphic and pathological infarct size was observed in infarction and myocarditis. ${ }^{12}$ This method accurately detected myocardial infarction, ${ }^{10} 13$ cardiac transplant rejection, ${ }^{14} 15$ and myocarditis in humans. ${ }^{16} 17$ In our patient, in spite of active muscular disease, there was no uptake by skeletal muscle. The positivity of the antimyosin scan suggests the existence of myocytic necrosis, one of the basic lesions of acute myocardial disease in polymyositis. In myocarditis, normal coronary arteries give a good signal to noise ratio, even if the number of necrotic cells is not as high as in an acute myocardial infarction. Normalisation of both myocardial scintigrams (thallium and antimyosin) after treatment, with a favourable clinical evolution of the polymyositis, confirms the specificity of the abnormalities seen initially in this patient.

Cardiac disease in polymyositis/dermatomyositis is an indicator of a severe disease state and its incidence is probably underestimated. The management of these patients should include evaluation and follow up of their cardiac status. This case suggests that 
myocardial antimyosin antibody scintigraphy may be a helpful, safe, and more specific tool than conventional non-invasive techniques for the diagnosis and follow up of acute myocardial disease in polymyositis. Further studies are needed to determine its accuracy and to specify which patients can benefit from this technique.

1 Bohan A, Peter J B, Bowman R L, Pearson C M. A computer-assisted analysis of 153 patients with polymyositis and dermatomyositis. Medicine (Baltimore) 1977; 56: $255-86$.

2 Askari A D, Huettner T L. Cardiac abnormalities in polymyositis/dermatomyositis. Semin Arthritis Rheum 1982; 12: 208-19.

3 Hochberg M C, Feldman D, Stevens M B. Adult onset polymyositis/dermatomyositis: an analysis of clinical and laboratory features and survival in 76 patients with a review of the literature. Semin Arthritis Rheum 1986; 15: 168-71.

4 Gottdiener J S, Sherber H S, Hawley R J, Engel W K. Cardiac manifestations in polymyositis. $\mathrm{Am} \mathrm{f}$ Cardiol 1978; 41: 1141-9.

5 Henrikkson K G, Standstedt P. Polymyositis-treatment and prognosis: a study of 107 patients. Acta Neurol Scand and prognosis: a study

6 Larca L J, Coppola J T, Honig S. Creatine kinase MB isoenzyme in dermatomyositis: a noncardiac source. Ann Intern Med 1981; 94: 341-3.

7 Denbow C E, Lie J T, Tancredi R G, et al. Cardiac involvement in polymyositis: a clinicopathologic study of 20 autopsied patients. Arthritis Rheum 1979; 22: 1088-92.
8 Haupt H M, Hutchins G M. The heart and cardiac conduction system in polymyositis-dermatomyositis: clinicopathologic study of 16 autopsied patients. $A m$ Cardiol 1982; 50: 998-1002.

9 Plotz PH, Dalakas M, Leff R L, Love L A, Miller F W, Cronin M E. NIH conference: current concepts in the idiopathic inflammatory myopathies: polymyositis, dermatomyositis, and related disorders. Ann Intern Med 1989; 111: 143-57.

10 Khaw B A, Yasuda T, Gold $\mathrm{H} \mathrm{K}$, et al. Scintigraphic quantification of myocardial necrosis in patients after intravenous injection of myosin-specific antibody Circulation 1986; 74: 501-8.

11 Khaw B A, Fallon J T, Beller G A, Haber E. Specificity of localization of myosin specific antibody fragments in experimental myocardial infarction: histological, histochemical, autoradiographic and scintigraphic studies. Circulation 1979; 60: 1527-31.

12 Johnson L L, Lerrick K S, Coromilas J, et al. Measurement of infarct size and percentage of myocardium infarcted in a dog preparation with single photon emission computed tomography, thallium-201 and indium 111-monoclonal antimyosin Fab. Circulation 1987; 76: 181-90.

13 Khaw B A, Yasuda T, Gold, H K, et al. Acute myocardial infarct imaging with indium-111-labeled monoclonal antimyosin Fab. $\mathcal{F}$ Nucl Med 1987; 28: 1671-8.

14 Frist W, Yasuda T, Segal G, et al. Non-invasive detection of human cardiac transplant rejection with indium 111 antimyosin (Fab) imaging. Circulation 1987; 76 (V): $81-5$.

15 Ballester-Rodes M, Carrio-Gasset I, Abadai-Berini I Obrador-Mayol D, Berna-Roqueta L, Caralps-Riera J M. Patterns of evolution of myocyte damage after human heart transplantation detected by indium 111 monoclonal antimyosin Fab. Am $\mathcal{F}$ Cardiol 1988; 62: 623-7.

16 Khaw B A, Scott J, Fallon J T, Cahill S L, Haber E, Homcy C. Myocardial injury: quantification by cell sorting initiated with antimyosin fluorescent spheres. Science 1982; 217: 1050.

17 Yasuda T, Palacios I F, Dec G W, et al. Indium 111 monoclonal antimyosin antibody imaging in the diagnosis of acute myocarditis. Circulation 1987; 76: 306-11. 